\title{
Métaphorisations mutuelles, mise en scene et médias : invitations à l'induction?
}

\section{Wolfgang Settekorn}

\section{(2) OpenEdition}

1 Journals

Édition électronique

URL : http://journals.openedition.org/communicationorganisation/1990

DOI : 10.4000/communicationorganisation. 1990

ISSN : $1775-3546$

Éditeur

Presses universitaires de Bordeaux

\section{Édition imprimée}

Date de publication : 1 novembre 1997

ISSN : 1168-5549

\section{Référence électronique}

Wolfgang Settekorn, « Métaphorisations mutuelles, mise en scene et médias : invitations à

l'induction ? ", Communication et organisation [En ligne], 12 | 1997, mis en ligne le 26 mars 2012,

consulté le 03 mai 2019. URL : http://journals.openedition.org/communicationorganisation/1990 ;

DOI : 10.4000/communicationorganisation. 1990

Ce document a été généré automatiquement le 3 mai 2019.

(c) Presses universitaires de Bordeaux 


\title{
Métaphorisations mutuelles, mise en scene et médias : invitations à l'induction?
}

\author{
Wolfgang Settekorn
}

\section{Métaphores et conceptualisation}

1 Ce que nous entendons par « communication » dépend en grande partie des métaphores que nous utilisons lorsque nous parlons de ce phénomène complexe. En effet, une fonction essentielle des métaphores tient dans le fait qu'elles nous permettent de concevoir des faits complexes sous forme réduite. Pourtant, on constate une diversité de termes et de concepts métaphoriques existant pour décrire un même fait. Cette diversité résulte d'une part des différents aspects factuels qui ne peuvent pas être réduits par une métaphore unique, et d'autre part des aspects spécifiques qui y sont thématisés. Klaus Krippendorf (1990) a montré dans quelle mesure ceci est également valable pour les termes de communication quotidiens et scientifiques.

2 Mais qu'entendons-nous par «métaphores"? Je m'en tiens à Lakoff et Johnson qui définissent les métaphores comme suit: "The essence of a metaphor is understanding and experiencing one kind of things in terms of another » (Lakoff/Johnson 1980, 5). Dans cette théorie, les métaphores ont un domaine source et un domaine cible. Les éléments du domaine source peuvent être reportés sur le domaine cible par un "source-to-domainmapping " (Lakoff 1987, 276). Lakoff rend ce processus explicite en utilisant l'exemple de MORE IS UR LESS IS DOWN. Ils s'en réfère à des expressions telles que : "The crime rate keeps rising. The number of books published each year keeps going up. The stock has fallen again. Our sales dropped last year. You'll get a higher interest rate with them. Our financial reserves couldn't be any lower » (Lakoff 1987, 276). La VERTICALITÉ constitue ici le domaine source «consciously, and without noticeable effort as part of normal functioning. Concepts used in this way have a different, and more important, 
psychological status than those that are only thought about consciously " (Lakoff 1987, 12).

3 Lors des préparatifs de la guerre du Golfe Lakoff a montré, dans "Metaphor and war " que l'utilisation et l'adoption de métaphores spécifiques dans des situations concrètes pouvaient transporter des visions du monde et entraîner des décisions politiques. Dans ce processus, les mass-médias ont joué un rôle important et peu glorieux qui a été analysé maintes fois. Les informations étouffées ou encore leur mise en circulation savamment manipulée dans le cas présent ont montré de manière particulièrement claire que nous acquérons nos connaissances par voie d'une expérience médiatique. Au-delà de ce cas, il faut se demander de façon générale quel rôle joue la présentation médiatique dans la mise en concept et particulièrement dans l'incorporation fonctionnelle de circonstances et de domaines ayant trait à la vie. Les réflexions qui suivent reposent sur l'hypothèse que la mise en scène d'événements souvent auto-initiée et réalisée par les mass-médias eux-mêmes, ainsi que le mode de leur présentation médiatique, contribuent pour beaucoup à la constitution automatique, inconsciente et sans grands frais de concepts qui y sont utilisés. Je pars du principe qu'il s'agit là de concepts qui sont partagés par beaucoup, sinon par la plupart des membres d'une culture.

4 Compte-tenu du manque d'images sur le déroulement de la guerre, les médias audiovisuels se célébraient eux-mêmes dans leurs informations sur la guerre du Golfe : leur façon de rapporter les faits était en grande partie auto-référentielle et montrait que le représentants des différentes chaînes, s'ils n'étaient pas sur place, étaient tout au moins proches des événements et que les connexions par satellites entre studios, émetteurs et reporters fonctionnaient. Il s'agissait là d'un triomphe de l'interaction phatique et de la métacommunication auto-référentielle.

5 Même s'ils ne sont pas toujours aussi clairs que dans ce cas, les processus autoréférentiels ont une place importante à la télévision. J'ai signalé en d'autres lieux leur fonction dans les séquences d'introduction des derniers débats de la campagne présidentielle française (Settekorn 1989, 1990). Il en ressortait qu'une expression telle que "À de nombreuses reprises, surtout sur la scène internationale, on vous a vus, l'un et l'autre, assez proches » (Settekorn 1989, 42)

- englobait le spectateur par le pronom «on »

- se basait à travers la forme verbale (on vous a vus) sur des événements observés dans le passé et,

8 - en même temps, sur l'événement visuel actuel par le verbe (les spectateurs voient ce que dit Michèle Cotta)

9 - qu'elle contenait une fonction auto-référentielle en vue de laquelle

10 - l'expression métaphorique de la "scène internationale " perd une partie de son caractère métaphorique ; elle est concrétisée par voie de référence aux circonstances générales du mode de perception actuel.

11 L'écran montre ce qui se passe sur la scène internationale; c'est à travers lui, et en général uniquement à travers lui qu'elle est accessible aux spectateurs. L'écran devient ainsi le lieu de présentation de la scène internationale, pour ne pas dire qu'il coïncide même avec elle. Les circonstances d'utilisation de la métaphore correspondent ici à des éléments essentiels du domaine source : ce dont on parle avec une métaphore correspond ici à une situation d'utilisation, dans laquelle des éléments essentiels de la signification «littérale » sont pratiquement repérables. Ils concordent donc en ce sens et de manière spécifique avec la 
réalité, ce qui permet d'effectuer une (auto-) vérification pragmatique. Je suppose que des cas à ce point auto-référentiels conviennent particulièrement pour contribuer à la formation d'une incorporation fonctionnelle. La situation d'utilisation et le domaine source correspondent ensemble et rendent ainsi compréhensible in actu la situation décrite de façon métaphorique. Ceci vaut également dans le cas de la métaphorisation bilatérale qui doit être au centre des réflexions qui suivent.

\section{Métaphorisation mutuelle (bilatérale)}

Que faut-il entendre par métaphore mutuelle ou bilatérale? Dans la conception de la métaphore décrite ci-dessus, la transmission des attributs est orientée de façon unidirectionnelle des domaines source vers les domaines cible. Le domaine cible est pour ainsi dire conceptualisé à partir du domaine source. Je voudrais attirer l'attention sur un phénomène, dont le développement s'est accéléré, si je vois juste, ces derniers temps avec l'influence du développement des médias. Il s'agit de la métaphorisation mutuelle de domaines qui est pratiquée de plus en plus intensément dans les discours de mass-médias. Les domaines sources et les domaines cibles y sont utilisés par substitution mutuelle.

Avec ce rapport, qui reste à montrer, apparaissent donc non seulement des conceptualisations spécifiques et des visions du monde, mais aussi des changements de pratiques sociales et de perception.

Sport et économie sont les deux domaines, dans lesquels les processus de métaphorisation mutuelle sont particulièrement appréciés. Cela n'est pas étonnant dans le sens sportéconomie, compte-tenu de la longue tradition qui consiste à utiliser métaphores et métonymies, issues du domaine sportif et de la compétition, pour illustrer d'autres domaines de la vie sociale. La politique et l'économie font ici tout aussi peu exception que la communication, la culture, l'éducation et la science.

L'emploi de techniques identiques ou semblables dans la présentation médiatique établit une partie du travail vers la comparabilité et l'homogénisation. Pensons, par exemple, à la possibilité de rendre la position actuelle d'un élément isolé calculable en un coup d'œil, en transformant en chiffres dans les domaines les plus différents, la situation respective d'un domaine et de ses éléments, grâce à des tableaux basés sur des résultats. Cela nécessite également des diagrammes d'évolution qui, au-delà de la période choisie, rendent l'évolution représentable selon des critères définis. Peu importe s'il s'agit de celle d'une entreprise, d'une équipe ou d'un individu. En plus de ces conditions techniques et médiatiques générales qui ont été rapidement améliorées par l'introduction renforcée de l'infographie et par des logiciels abordables et faciles d'utilisation, apparaît un paysage médiatique européen changé en profondeur et qui a lui-même été commercialisé par la dérégulation de la radio et de la télévision. C'est ainsi que le poids de la forme et du contenu des programmes se sont transformés. Le fait que les présentateurs annoncent les coupures publicitaires à l'aide de la remarque déjà stéréotypée dans la télé allemande « et maintenant, il faut que nous gagnons un peu d'argent » est dans ce cas devenu un indice qui ne nous frappe pratiquement plus.

\section{Le sport : aimant à spectateurs et marchandise}

Le sport et, en dehors du tennis le football en particulier, est devenu en tant qu'outil pour attirer le spectateur une marchandise, dont la valeur marchande n'a cessé d'augmenter. 
Ceci étant, le caractère de marchandise transforme le sport, y compris dans ses formes, ses structures et ses représentations. Le fait que fin 1996, en Allemagne, la proposition de RTL et SAT 1 ait été discutée de manière controversée et avec beaucoup d'engagement, n'est qu'un indice des transformations profondes du sport et des informations sportives. Selon cette proposition, le déroulement des matchs de football retransmis (et pas seulement de ceux-là) doit être modifié de telle façon que les chaînes puissent gagner, grâce à plus de pauses publicitaires, l'argent qu'elles ont dû débourser après un rude combat avec la concurrence pour obtenir les droits de retransmission. Il semble qu'aux yeux des programmateurs de chaînes privées, les programmes ne puissent être rentables sans cette modification.

\section{L'économie dans la rubrique « sport »}

Faut-il s'étonner dans ce contexte que les informations sportives prennent bien souvent l'allure d'informations économiques. On y traite déjà depuis longtemps le fait qu'après le jugement de Bosmann, les clubs aient à faire face à des problèmes s'ils ne peuvent plus compter sur les recettes de transfert prévues. Il y est tout autant question du fait que les joueurs achetés pour de grosses sommes perdent de la valeur en un tour de manivelle à travers leurs performances médiocres ou suite à des blessures, et que d'autres, au contraire, la multiplient en quelques semaines en tant par exemple, que gardien de but couronné de succès. Tout cela, nous l'entendons dans les informations sportives, tout comme l'expression disant d'un joueur qu'il s'avère être un bon investissement et d'un autre qu'il est une grande déception. Nous ne nous rendons pratiquement plus compte que des personnes sont devenues des marchandises et des produits dont la durée de conservation est courte. En effet, contrairement aux bilans économiques de quelques clubs, cet aspect particulier est traité extrêmement rarement, sinon jamais.

En même temps, le statut des sportifs de haut niveau a changé. Nous connaissons depuis des années des sportifs de haut niveau en tant que stars de l'industrie de la publicité convoités et hautement rétribués (Faulstich 1991). La possibilité d'assurer de telles ressources fait souvent partie intégrante des contrats de travail signés avec les clubs. Après avoir été des amateurs ou des amateurs nationaux plus ou moins bien rémunérés, ils sont devenus des professionnels. Et il faut se poser la question de statut suivante et pas seulement pour les mégastars appréciées et préférées de la branche : «les footballeurs sont-ils des entrepreneurs ou des salariés? » (Hamburger Abendblatt 1996, 13, 12, page 26 «Sportteil »). Même si cette question doit finalement trouver une réponse juridique, les conséquences des faits ci-dessus exposés vont beaucoup plus loin. Elles concernent le changement profond auquel est soumis le sport dans la vaste transformation technologique, médiatique et économique des dernières années. Quelques exemples issus des informations sportives peuvent rendre les faits exposés plus clairs.

C'est ainsi que nous trouvons en pleine partie sportive un communiqué du 1. FC Kaiserlautern qui a quitté la première division avec le titre suivant :

Là-haut, sur le Betzenberg, il y a des millions dans le béton. Les nouveaux responsables du Kaiserslautern veulent à nouveau investir plus dans le sport et renversent l'ordre établi par les dettes.

21 Tout l'article se lit effectivement tel un bilan économique qui informe sur les aspects négatifs et positifs, sur les mauvaises anciennes stratégies et les nouvelles stratégies modifiées d'une entreprise. On apprend qui a acheté quoi pour combien et pour combien 
on a vendu. On apprend ce qu'il en est du compte bancaire du club et quelles sont les stratégies économiques du club. Et puis la fin de l'article récapitule ce que le sous-titre annonçait : il y était dit qu'on voulait « investir dans le sport ». La dernière phrase varie un peu : « Il vaut mieux investir à l'avenir dans des joueurs, de très bons joueurs tant qu'à faire ».

A travers de tels communiqués, on a l'impression que par la communication du sport, la situation économique des clubs est tellement passée au premier plan qu'il faille redécouvrir le côté sportif. Plus encore: même dans les comptes-rendus de match, l'aspect économique joue un rôle de plus en plus important, ce qu'il faudrait encore montrer en particulier.

\section{Rubrique économique et rubrique sportives dans certains quotidien}

L'article concernant la situation économique du 1. FCK se trouvait dans une partie sportive séparée de l'édition du lundi 4 novembre 1996. Comme pour tous les montages, on trouve le compte-rendu sportif de ce journal comme feuillet séparable qui informe sur les événements sportifs du week-end précédent. Le fait qu'il en soit ainsi rappelle une longue tradition au cours de laquelle le sport se déroulait pour la plupart des sportifs, des entraineurs et des moniteurs, mais plus encore pour les spectateurs, lors des loisirs et principalement sur les lieux de l'événement: sur les stades, les hippodromes, les gymnases et dans les piscines. Surtout pour les spectateurs, les événements sportifs avaient lieu le week-end, en dehors de la sphère professionnelle. Les activités de la semaine servaient surtout à l'entraînement et à la préparation de ces mêmes événements. Cette répartition changea avec la dérégulation des médias électroniques et leur privatisation: l'internationalisation des compétitions et la fixation sur de soi-disant, ou de véritables, équipes de haut niveau encouragèrent la spéculation et l'attraction des événements pour l'information télévisée. Le cycle des événements fut (de plus en plus souvent) accéléré et (de plus en plus) condensé. Les chaînes spécialisées relatent toute l'année et 24 heures sur 24 les actualités sportives.

La perspective de ressources encore extensibles (de plus en plus de spectateurs payant dans les stades et payant aussi la redevance télévision) exigea de beaucoup investir et à long terme. Le sport devint donc partout un commerce et un métier dont beaucoup vivent et dont les différentes parties attendent non seulement un profit symbolique plus élevé, mais surtout un gain économique. Et ce n'est pas tout. La structure et le statut des clubs changent aussi : les fonctionnaires du temps libre bénévoles et non rémunérés (souvent présentés comme des maladroits et des bâcleurs) sont remplacés par des professionnels et des managers (on ne parle, du côté des clubs et des fédérations, pourtant guère volontiers de leurs faillites et de leurs pannes). Les clubs déclarés sont en pleine mutation pour devenir des entreprises économiques et des sociétés anonymes.

\section{L'économie avant le sport}

Quoi d'étonnant dans ces conditions que le sport soit situé en semaine dans beaucoup de quotidiens, tels Hamburger Abendblatt, Frankfurter Allgemeine Zeitung et Frankfurter Rundschau dans le même feuillet que la partie économique. La proximité créée par le 
layout est tout sauf une coïncidence. En parcourant les pages, elles deviennent palpables et proches dans le sens. Cela contribue lors de la lecture à un sentiment d'incorporation, d'«embodiment " au sens de Lakoff. Un premier niveau de proximité médiatique des sens est présent ici dans le cas de la liaison entre sport et économie, si on parle d'induction comme mode propre à la communication.

Si on part du principe que les habitudes de lecture occidentales vont de la gauche vers la droite et d'autre part que les mass-médias pratiquent habituellement la mise en avant spatio-temporelle des faits importants et marquants, on en arrive à une constatation supplémentaire : en règle générale, la rubrique sportive est, dans les exemples cités, «le pendeloque » - si on veut le dire de manière négative pour le sport - ou - pour tenter de le dire de façon plus neutre - une "suite » de la rubrique économique. En tout état de cause, il lui succède. On pourrait, dans ce cas, dire «le sport succède à l'économie » ou «l'économie précède le sport ».

De nombreux exemples, qui vont du nom de l'entreprise et du logo imprimé sur les vêtements des entraîneurs et joueurs, en passant par les tricots et les publicités en banderole, jusqu'à « l'image complète » des clubs et fédérations, montrent que ceci est valable dans le sens propre comme dans le sens figuré. C'est ainsi que l'équipe nationale de football allemande joua lors de la coupe d'Europe 1996 « sous une bonne étoile » (celle de Mercedes). Le cas de cet ancien joueur de l'équipe nationale allemande qui réussit, lors de la coupe d'Europe dans son commentaire de match et en conversation avec un reporter sportif d'une chaîne d'état, à faire abondamment de réclame pour l'entreprise qu'il représentait, montra clairement que la langue, et surtout celle des reporters de chaînes privées, mais pas seulement, suit cette tendance.

\section{Sport- Economie- Médias}

Le fait que le sport suive l'économie est, en ce qui concerne les médias, suffisamment confirmé par la pratique des reportages sportifs. Pourtant, il ne s'agit pas là d'une simple relation transitive, l'inversion est également valable : l'économie est à la suite du sport et des médias. Le sens du sponsoring tient dans le do ut des, c'est à dire dans le fait de mentionner le sponsor, de faire connaitre son nom, d'établir et d'élargir son degré de connaissance dans le public, et donc dans la " public relation ».

Cela conduit à la possibilité de métaphorisation mutuelle dans la présentation et l'interprétation d'événements, de projets et d'agissements et en même temps à celle de voir des agissements sous un angle spécifique et de donner des modèles pour les déroulements d'événements plus complexes. Le journal Welt am Sonntag du 3 novembre 1996 montre à quoi cela peut ressembler avec ses rubriques rassemblées dans le feuillet «Placement de capitaux-TV ». Elles allaient des pages 63 à 76 (cf. nº 1).

\section{La « première division des bourses »}

30 Les premières pages de cette partie servaient à introduire la première division à la bourse. Sur la première page de cette partie, la première grande manchette imprimée en gras apporte la citation à première vue étonnante :

«Les valeurs du sport devraient être valables dans l'économie ». 

Première division des bourses de Wams qui se révèle être sur la page suivante également le jeu de pronostics de la première division des bourses de Wams. En même temps, l'organe de presse se comporte comme ces clubs sportifs, organisateurs culturels ou ces émissions télévisées qui rendent public le soutien amical d'un sponsor, dont le nom est cité. L'organe le fait ici en accord mutuel et avec les mêmes attentes qui sont d'élever le profit financier et symbolique au maximum.

\section{Présentation de l'interview}

Dans une petite ligne postée au-dessus et relativement longue dans le layout, on apprend qui en est l'auteur :

Manfred Maus, fondateur et sociétaire gestionnaire du système de franchise OBI, magasins de construction et de bricolage.

$\mathrm{Au}$ milieu de la première partie de l'interview qui compte cinq colonnes, on trouve un cadre dans lequel, en utilisant de manière ciblée les couleurs bleu, blanc, noir et rouge, a été placé le logo de la nouvelle que Welt am Sonntag et OBI ont choisi d'annoncer dans le monde des médias et au public (cf. $\left.n^{\circ} 2\right)$ :

WELT am SONNTAG

Première division des bourses avec OBI

Derrière OBI, on voit la mascotte de l'entreprise riant et levant le pouce droit vers le haut. Le cadre entoure l'article comme la coquille enveloppe le noyau.

colonnes. Le titre principal y est répété. Au milieu de cette vue, on a placé une photo de l'interview (cf. $n^{\circ} 3$ ) (sous-texte : le rédacteur de Wams Ulric Papendick (à gauche) et Joachim Reuter avec Manfred Maus).

L'organe de presse peut donc être pratiquement sûr que grâce à son apparition répétée avant les émissions sportives et publicitaires, l'animal du logo est bien connu, en tant que mascotte de son entreprise, par le lecteur qui regarde également la télévision. Comme le nom de l'entreprise suivi de l'animal est plus connu en tant que signe de la marque que le chef de l'entreprise - il était pour beaucoup plutôt un Maus grisâtre -, on peut penser que sa présentation détaillée en image et en parole, ainsi que le fait qu'il prenne la parole dans une interview si importante, servent non seulement à promouvoir la nouvelle à annoncer, mais aussi à assurer sa promotion personnelle et publique.

Le logo de la première division des bourses ainsi présenté a pour sous-texte :

Jeu de la bourse de Wams : Infos sur la page suivante

Et ainsi saute aux yeux ce que l'interview exprime plus en détails : Obi sponsorise la

La remarque préliminaire à l'interview rend sans ambiguïté la mise en parallèle effectuée entre sport, économie et finances à l'aide du terme métaphorique :

«C'est aujourd'hui qu'est donné le signal de départ de la première division des bourses ».

C'est de cette façon qu'est formulée la première phrase. L'utilisation de l'article défini contenu dans « de la » suggère que le fait est connu et la formule métaphorique issue du domaine sportif parlant de «signal de départ» fait disparaître les questions supplémentaires comme si cela était évident : Qui tire, où et quand exactement? 
Celui qui pose de telles questions se révèle être un ignorant en regard de la campagne publicitaire dense, débutée il y a longtemps et dans tous les médias pour préparer la phase actuelle et de grande actualité qui concerne l'introduction de l'action Télécom. Et il doit l'être, car il n'est pas question que naisse un scepticisme à l'égard de cette gigantesque transformation de la poste nationale, méticuleusement disséquée auparavant en entreprises partielles. Ce scepticisme saurait se répandre quant au succès du domaine de la télécommunication, en pleine mutation pour devenir une société anonyme. Il y a quelque chose dans l'air dans le climat créé par cette campagne et le journal Welt am Sonntag fourre son nez en plein dedans. C'est tout au moins de cette façon que la phrase suivante de la remarque préliminaire l'annonce :

Welt am Sonntag s'est entretenu avec le chef de Obi, Manfred Maus, sur les actions et le capital à risque, la nouvelle culture du placement de capitaux et également sur les raisons qui le poussent à soutenir le jeu de la bourse.

48 Le domaine mis en gros et en évidence dans la manchette est placé à la fin, comme annoncé ci-dessus. Celui qui ne sait pas encore ce qu'il en est de la Première division des bourses doit tout d'abord prendre connaissance des renseignements spécialisés de la personne compétente interviewée, dont il faudra attendre la prise de parole sur la nouvelle culture de ceux qui placent des capitaux avec plus ou moins d'impatience. Maus se transforme ainsi, sans avoir l'air d'y toucher, en un potentiel, ou un véritable, créateur de culture du placement de capitaux. On n'apprend (tout d'abord) rien sur lui-même et son entreprise et surtout rien de critique, puisqu'il faut être « tout dans Obi » et le rester.

\section{L'image de l'économie et du monde selon Monsieur Maus}

49 Les intervieweurs préalablement nommés, ont confiance dès leur première question dans le fait que ce dont on parle ou, plus exactement même, ce dont on laisse parler dans les médias et surtout dans la réclame, est également connu des lecteurs. Ils mènent l'odeur que l'actuelle campagne laisse circuler vers le nez de l'interviewé et du lecteur:

Welt am Sonntag: Monsieur Maus, les mots " bourse " et " action " sont actuellement dans toutes les bouches. Les Télécoms veulent enthousiasmer une grande partie de la population pour que les gens deviennent actionnaires. Que signifient pour vous ces mots à la mode?

1 « Mots à la mode » n'est pas à prendre ici dans le sens négatif, mais plutôt dans les sens de «mots-clés». C'est de cette façon que la personne interviewée le comprend également puisqu'elle trace les contours de sa vision du monde et de son opinion à l'aide de mots de code.

52 Maus: Dans ces deux termes, il est question de gagner de l'argent, sachant que risque et chance sont très près l'un de l'autre. Le monde est en train de changer, les hommes ont plus de moyens et placent leur argent aujourd'hui différemment. Le changement de valeurs fondamental de notre société a pour conséquence que les gens se tournent vers les actions. Il s'agit là de quelque chose de nouveau.

53 Ce qu'on dit là semble si clair et si évident: Maus sait ce qu'il veut et semble également savoir ce qu'il dit. La première moitié de phrase amène la réponse dans la direction souhaitée. Dans un article de la rubrique "Placement d'argent », on attend dès le départ qu'il s'agisse de gagner de l'argent lorsqu'on parle de bourse et d'action. Mais s'agit-il vraiment de "GAGNER de l'argent », et qui gagne son argent quand et avec quoi ? De qui parle-t-on? Du boursier, de l'agent de change, des boursiers, des petits porteurs, des 
imprimeurs d'actions, des Télécoms qui se font payer les lignes téléphoniques de la bourse ou bien qui, et qui encore?

Il semble certes que la deuxième moitié de la phrase isole le domaine de ceux à qui Monsieur Maus peut bien vouloir penser, mais il semble malgré tout très imprécis : qui a le risque, qui en est responsable et qui encourt quel risque ; qui a les chances énoncées? On n'apprend rien de précis et visiblement, on ne doit rien apprendre. C'est en tout cas ce que montre la phrase suivante à travers sa généralisation déjà presque sans limites et son absence de limites. La constatation de ce que le monde est en train de changer est quelconque et intemporelle, si bien qu'elle n'offre rien de plus qu'une formule toute faite, utilisée comme stratégie de discussion et qui ne peut que difficilement être contredite en vue de sa généralité. Mais qu'est-ce-que cela peut bien être "le monde»? Seule une chose est sûre, Dieu manque et donc il ne peut être question de "Dieu et le monde ». Il ne s'agit certainement pas non plus de l'édition dominicale de l'organe qui a mené et imprimé l'interview, dans la mesure où ce monde ne change pas tant que cela.

De cette constatation générale se dégage pour Maus et ses lecteurs l'effet particulier suivant: Faut-il dire, on arrive à la fortune par le changement du monde ou alors le changement du monde mène à la fortune. Monsieur Maus ne le dit pas de façon aussi simple et dans le cas présent, il est déjà un peu plus précis, mais reste à la fois à nouveau terriblement général : « les hommes ont de plus en plus de moyens ». Vraiment, on lit: LES hommes.

\section{Maus et les hommes}

On sait maintenant grâce à l'analyse de l'utilisation des médias que les rubriques spécialisées ne sont pratiquement lues que par les personnes intéressées par le sujet et que les autres les sautent tout simplement. Cela a pour conséquence que la portée des quantificateurs et déterminateurs pragmatiques doit être fixée. Ce que cela signifie se laisse également illustrer - restons dans le sujet - par un exemple tiré du sport : quand on lit dans un magazine publié par un club de football les pronoms "nous » et "nos ", on sait, grâce à la situation, qu'il s'agit du club local ou de l'équipe locale. S'il s'agissait de l'équipe adverse, il faudrait l'exprimer clairement.

Mais monsieur Maus ne parle pas expressément de « nous », bien qu'il soit possible que ce soit ce qu'il veut dire. Il parle de «LES », et par là même de la possibilité au moins qu'il s'agisse de tous les hommes. Dans le sens littéral, il se pourrait qu'on parle de toute l'humanité, surtout que celle-ci est soumise au changement du monde précédemment évoqué. Bien sûr, Monsieur Maus ne veut pas dire cela, mais que veut-il donc dire?

Nous savons qu'au cours des dix dernières années - afin de donner une limite temporelle à ce changement du monde - quelques personnes se sont enrichies dans les anciens et dans les nouveaux Länder. Cette constatation rebattue est devenue une notion générale et derrière la formule de Monsieur Maus se cache autre chose. Pour ne pas laisser apparaître dans les esprits et les bouches le mot de société des deux tiers qui ne fait pas son affaire, il fait de la partie riche de la société, donc du premier tiers, LES hommes en général. Et il passe les deux tiers défaillants. Ceci correspond à la vision d'un entrepreneur au niveau de la réalité sociale et en même temps au sujet dont il est débattu: les gens à moindres revenus ne font pas d'achats dans les boutiques de luxe et ne s'occupent pas de la bourse et des actions. Ils ont ce qu'ils méritent, mais justement pas de l'argent, tel que Monsieur Maus le pense, dans la mesure où ils ne le laissent pas dans ses magasins. Et pourtant, il y 
amènent une partie de leurs revenus lorsqu'ils vont se procurer chez lui quelque chose pour bricoler. Et si ils ont acheté leur matériel auparavant chez un petit commerçant ou dans une autre chaîne de magasins de construction, alors « ils » placent « aujourd'hui » leur argent autrement qu'avant, et pour certains d'entre eux, ils placent à ses yeux peutêtre même une fortune.

Monsieur Maus ne parle pas d'eux dans l'interview dont il est question, mais de ceux qui ont, d'une façon ou d'une autre, suffisamment d'argent et une fortune qui a suffisamment augmenté. Ceux-là peuvent maintenant placer leur argent dans des actions. Tout cela est, d'après Monsieur Maus, à mettre sur le compte d'un changement fondamental des valeurs. Les conséquences en sont que «les hommes [ici encore] se tournent vers les actions ». Heureux soit celui qui peut se faire accorder cette attention.

\section{Économie, valeurs et choix de mots}

Monsieur Maus parle ici, peut-être même sans le savoir, d'un air apostolique : L'argent, les actions et la propriété sont Dieu, la bourse est son temple et Maus en arrive à être le prophète d'un nouveau monde fondamentalement changé. Le changement de valeurs annoncé en 1982 a été accompli d'une manière évidente après quatorze ans de gouvernement démocrate-chrétien et libéral et Maus l'annonce du point de vue de l'entrepreneur et avec une diction qui rappelle celle du Nouveau Testament. La jeunesse avait, grâce à une meilleure formation, un « rapport différent envers les biens ». À l'aide de nouvelles formes de capitalisation, il faut aider « les jeunes entreprises » pour réaliser la « culture du capital à risque » que demandent certains (lesquels ?) politiciens.

\section{Complicité dans l'introduction de la « première division des bourses »}

61 Dans un tel contexte, le jeu de la bourse doit apporter sa contribution positive. Le reste du contexte rédactionnel informe de manière détaillée sur les modalités de ce produit dernier cri, issu de l'ingéniosité de l'économie et des médias. Tout comme dans le concours de pronostics, les participants doivent prévoir, sur un «billet de pronostic pour la série de jeu n ${ }^{\circ} 1 »$, les résultats de jeu de neuf couples d'entreprises et/ou envoyer en payant $20 \mathrm{DM}$ la «demande d'ouverture d'un dépôt». On trouve sur la page 64 un exemplaire des deux formulaires qui portent le même titre "première division des bourses - WELT am SONNTAG - OBI ». Tout cela doit attirer, par le jeu, l'intérêt d'un large public pour la bourse et donc offrir à cette nouvelle culture le milieu financier nécessaire.

La façon dont Maus parle de tout cela et dont il évoque comme si de rien n'était son entreprise, montre clairement le rapport entre économie, médias et sport :

La première division de bourses que nous menons ensemble est un de ces éléments pour aborder une large population : intéresse-toi à la bourse.

La première division des bourses sert donc de promotion générale de la bourse.

C'est pour cela qu'OBI soutient le jeu. Car en tant qu'entreprise de franchising, nous avons besoin d'un esprit d'entreprise pour mener les gens à l'autonomie.

Cela ressemble à une nouvelle philosophie des lumières et de la libération de l'homme (Une fois de plus de l'homme) d'un emprisonnement (dont il est lui-même responsable ?). Cela n'a pas seulement l'air philosophique, mais doit être philosophique, comme le dit Maus lui même dans la phrase suivante : 
professionnels, selon les avis, ainsi que leur organe se soient laissés appâter par les rondeurs du sponsoring de monsieur Maus. On le voit au logo et la façon de poser les questions le montre clairement à ceux qui lisent l'interview d'un œil critique. Une telle critique échappe totalement aux deux journalistes qui n'accordent aucune valeur à la différenciation et à la précision. Cela ne joue aucun rôle que Monsieur Maus n'ait pas «sponsorisé les jeux olympiques » et que Coca-cola, soit dit en passant, y apportait sa participation aussi. Le principal, c'est que Maus soit jeté à travers l'interview sous les feux de la rampe.

\section{Stratégies sémantiques et rhétoriques}

Maus a recours dans sa dernière réponse à son droit de définition et sait ce qu'est le sport:

«Quand on définit le sport, on ne peut passer à côté du terme performance. Vous devez être meilleur que le concurrent, apporter des résultats de haut niveau. Cela ne peut fonctionner que si je compare mes performances. J'ai besoin pour cela d'un terrain d'action adéquat, comme par exemple une première division, où les uns jouent contre les autres. Dans le cas présent, il s'agit de la première division des professionnels de la finance. Il y a des vainqueurs et des perdants exactement comme dans le sport » (mise en relief W.S.).

La métaphore prolongée de la première partie de réponse saute aux yeux: sport, compétition, vainqueur, perdant, profit, terrain sont les principaux termes 
correspondants. L'utilisation des pronoms personnels, avec lesquels Maus introduit dans son discours ces termes est pourtant plus intéressante que cette constatation. Pour les rendre plus évidentes, je les ai mises en gras. On sait qui parle, mais par contre on ne sait pas de qui Maus parle : de lui, de lui et des deux journalistes, en incluant les lecteurs ou non. Peut-être une fois de plus «des hommes", il les a déjà suffisamment souvent évoqués ? Parle-t-il donc de tout le monde ? Qui est « on », « vous », « je »?

$\mathrm{Au}$ premier abord, cette question peut sembler étrange. Si vous remplacez, chère lectrice ou cher lecteur, oui c'est à vous que je parle, vous à qui j'écris ces lignes, si donc vous remplacez dans le passage précédent «on» par «ils» ou «je » et exécutez les transformations grammaticales correspondantes, alors vous constaterez qu'au niveau du contenu, cel ne fait aucune différence, si Monsieur Maus dit « quand je définis le sport, je ne peux passer à côté du terme performance » ou « quand vous définissez le sport, vous ne pouvez passer à côté du terme performance » ou encore ce qui est imprimé dans le texte d'interview. Vous voyez, les pronoms de ce passage sont mutuellement échangeables.

78 Pourtant, au niveau rhétorique, leur séquence ne peut toutefois pas varier à volonté, car en dehors du fait qu'ils permettent d'éviter les répétitions, ils suggèrent un mouvement qui va aussi $\mathrm{du}$ " on » formel qui permet de caractériser de façon générale, au " vous » usuel qui est particulièrement distancé et poli jusqu'au « je », avec lequel un orateur parle lui-même. Il s'agit là d'une tendance qui a déjà parcouru toute l'interview. Elle suit la devise : l'organisation rhétorique ciblée passe avant la précision du contenu, ou alors : tout d'abord organisation rhétorique et ensuite précision du contenu (seulement si cela est nécessaire). Pour l'aménagement et la structure de l'article et de la campagne, il faut appliquer la devise: le design doit guider autant que faire se peut la conscience du « user ». C'est exactement sur cela que comptent les sponsors tels OBI et ils montrent rarement de façon aussi évidente que Monsieur Maus, qu'à travers les images télévisées, les métaphores sont mises en relation avec le monde et qu'à partir de là des points de vue et des valeurs doivent être transportés. Les sources de ces métaphores correspondent aux images qui y sont montrées et aux sponsors.

\section{Sponsoring et métaphores}

79 Le fait que ce terme puisse avoir un jour un sens littéral supplémentaire dans un paysage audio-visuel privatisé - ici, celui du sponsor qui procure les images que la télévision montre, et sur lesquelles se basent les métaphores avec lesquelles les sponsors veulent faire circuler les valeurs d'une économie transformée- tout cela était bien loin des esprits dans les années soixante-dix.

Monsieur Maus aborde justement cette situation transformée dans la partie suivante de sa dernière remarque :

81 «En sponsorisant les jeux olympiques, il était important pour moi, de faire passer les valeurs du monde sportif vers le monde de l'économie. Et ainsi, il faut que les concurrents soient respectés et qu'on ne pas construise pas d'inimitié. Le fair-play joue lui-aussi un grand rôle et on se passe du doping ".

82 Traduit dans notre langue cela signifie : il faut des prix loyaux pour le client. Nous avons formulé des modèles et l'un d'entre eux serait : savoir perdre avec bienséance. Si un jour, un client n'achète 
pas, il ne faut pas que je lui en veuille. C'est comme dans le sport. Je ne peux pas gagner chaque match. côté. Ce serait plus que déloyal de la part de Monsieur Maus d'exiger auprès de ces intervieweurs et ces questions d'aborder d'autres domaines. Les métaphores nettes et sa façon floue de parler, le contexte de l'interview et ses buts exprimés à l'aide d'images sportives, montrent pourtant ce qu'il veut voir et comment il veut voir, ainsi que ce qu'il ne veut pas voir. Il ne voit pas le chômage et la misère des postes dans l'enseignement, la distance entre des gains parfois effarants d'un côté et la montée du chômage et des faillites de l'autre. Il faut avoir le regard vissé sur les vainqueurs, les profits et la publicité ; et les médias sont bons compagnons. Eux aussi veulent leur morceau du gâteau du sponsoring, lorsqu'ils ne sont pas financés entièrement par la publicité. C'est ainsi que 
l'on pousse sur le devant de la scène ceux qui sont déjà sous la lumière. Les candidats à la descente et les perdants ne sont pas pris en considération, ils «jouent» dans les basfonds des amateurs en finance.

\section{Premier bilan}

Dans le circuit de l'économie, de la publicité des médias et du sport, le sport sert de métaphore à Maus pour faire circuler sa nouvelle vision de l'économie. Le sport devient dans ce jeu un double moyen de transport: d'un côté, il fait connaître le nom des sponsors en tant que bénéficiaire d'argent, d'un autre, il est domaine source de métaphores grâce auxquelles les médias et les sponsors peuvent faire passer leurs conceptions des objets dont il est question.

Dans la mesure où tout est, à travers sa présentation publique répétée, potentiellement compréhensible, la façon choisie pour présenter les choses rend clairs les contenus qui sont passés et cache le fait qu'il s'agit de changements profonds dans la société, l'économie et le sport. On nous cache aussi les mutations dont on peut lire les conséquences à d'autres chiffres. Les concepts utilisés par l'interviewé et son comportement dans l'interview en question donnent l'impression d'un pouvoir assuré par l'argent et la propriété. Maus le montre ouvertement. En même temps, il a appris la leçon de la campagne sémantique et conservative issue du temps du tournant moral et intellectuel.

89 Il est beaucoup trop évident que la comparaison entre le sport et l'économie vise à remplir les champs et les concepts. Et ici, la rhétorique des pronoms personnels est un moyen pour poursuivre une stratégie rhétorique qui part de l'acceptable général, et qu'on ne cherche pas à creuser, pour en venir au véritable sujet de discussion. En même temps, cette stratégie permet de ne pas s'engager sur une position individuelle grâce à la permutabilité des pronoms et pourrait être qualifiée de superficielle et égoïste.

Il est vrai que Maus joue un jeu plus facile que les politiciens de la CDU et de la CSU : la WELT n'est pas seule à être à ses pieds et à le soutenir, mais lui aussi, il soutient la WELT et prend part au sponsoring des jeux olympiques. Au contraire de ce qui se passe dans une campagne de propagande politique, cela n'échauffe pas les esprits, mais procure un certain prestige dans un public de plus en plus dépolitisé. Contrairement aux politiciens, les sponsors peuvent atteindre leur but à travers des médias, dans la mesure où même la structuration des événements traités est adaptée aux besoins des chaînes publiques et de leurs bailleurs de fonds. Un politicien ou un parti pourrait peut-être gagner du mérite aux yeux du public, s'il parvenait à donner un carton rouge à l'exigence d'un morcellement des événements sportifs adapté à la publicité. Mais pour cela, il faudrait peut-être que tous les partis politiques prennent des mesures. 


\section{BIBLIOGRAPHIE}

JOHNSON, Mark. The Body in the Mind: The Bodily Basis of Meaning, Imagination, and Reason. Chicago/ London : The University of Chicago Press, 1990.

KRIPPENDORF, Klaus. Der Verschwundene Bote. Metaphern und Modelle der Kommunikation. In : Medien und Kommunikation. Konstruktionen von Wirklichkeit. Studienbrief 3, Weinheim/ Basel : Beltz. S. 11-50,1990.

LAKOFF, John \& Mark JOHNSON. Metaphors We Live By. Chicago/London : The University of Chicago Press, 1980.

LAKOFF, John. Women, Fire, and Dangerous Things. What Vategories Reveal about the Mind. Chicago/ London : The University of Chicago Press, 1987.

SETTEKORN, Wolfgang. Politikinszenierung im französischen Fernsehen. Untersuchungen zu den Kandidatendebatten im Wahlkampf um die Präsidentschaft, in : HOLLY. W, KUHN. P, PUSCHEL (Hgg.) : Redeshows. Fernsehdi-skussionen in der Diskussion, Tübingen, 1l-47, 1989.

SETTEKORN, Wolfgang. «Le discours politique télévisé. Aspects de son analyse », Lendemains 59,115-123, 1990.

SETTEKORN, Wolfgang. « Rache der Natur », « Treibhauseffekt » und « Klimatote » - Von einfacher Rede über komplizierte Sachverhalte. In : Klima-Umwelt-Gesellschaft. Ein interdisziplinäres Seminar der Universität Hamburg, 16./17. November 1995, Hamburg, 70-83, 1996.

FAULSTICH, Werner. «Stras : Idole, Werbeträger Helden. Sozialer Wandel durch Medien ». Medien und Kommunikation, Konstruktionen von Wirklichkeit. Studienbegleitbrief 7. Weinheim/ Basel : Beltz, S. 39-79, 1991.

\section{AUTEUR}

\section{WOLFGANG SETTEKORN}

Université de Hambourg, Allemagne 